\title{
RNA viruses vs. DNA synthesis: a general viral strategy that may contribute to the protective antiviral effects of selenium
}

\author{
Ethan Will Taylor
}

Department of Chemistry and Biochemistry, The University of North Carolina at Greensboro Patricia A. Sullivan Science Building, PO Box 26170, Greensboro, NC 27402-6170, USA

\section{Correspondence: \\ Ethan Will Taylor \\ ewtaylor@uncg.edu}

\section{Keywords: RNA virus, DNA synthesis, selenium, thioredoxin reductase, SARS-coronavirus-2}

\begin{abstract}
The biosynthesis of DNA inherently competes with RNA synthesis because it depends on the reduction of ribonucleotides (RNA precursors) to 2'-deoxyribonucleotides by ribonucleotide reductase (RNR). Hence, RNA viruses can increase viral RNA production in cells by partially blocking the synthesis of DNA, e.g. by downregulating the mammalian selenoprotein thioredoxin reductase (TR), which normally acts to sustain DNA synthesis by regenerating reduced thioredoxin, a hydrogen donor for RNR. Computational and preliminary experimental evidence supports the hypothesis that a number of pathogenic RNA viruses, including HIV-1, Ebola, Zika, some flu viruses, and SARS-CoV-2, target TR isoforms by antisense. TR knockdown would create a host antioxidant defect that could be partially rectified by increased selenium intake, or be exacerbated by selenium deficiency, contributing to viral pathogenesis. There are several non-selenium-dependent means that viruses might also exploit to slow DNA synthesis, such as targeting RNR itself, or components of the glutaredoxin system, which serves as a backup redox system for RNR. HIV-1 substantially downregulates glutathione synthesis, so it interferes with both the thioredoxin and glutaredoxin systems. Computational results suggest that, like Ebola, SARS-CoV-2 targets TR3 by antisense. TR3 is the only TR isoform that includes an N-terminal glutaredoxin domain, so antisense knockdown of TR3 may also affect both redox systems, favoring RNA synthesis. In contrast, some DNA viruses encode their own glutaredoxins, thioredoxin-like proteins and even RNR homologues - so they are doing just the opposite, favoring DNA synthesis. This is clear evidence that viruses can benefit from shifting the RNA:DNA balance to their advantage.
\end{abstract}

\section{Introduction}

It is not a coincidence that the vast majority of the most notorious emerging and pandemic viruses, from the coronaviruses that cause SARS and COVID-19, to Ebola, HIV, avian influenza, Zika, Dengue, West Nile, Chikungunya, yellow fever, Eastern Equine Encephalitis, Norvirus, Nipah and Hantaviruses, as well as the less exotic measles, mumps, hepatitis viruses $\mathrm{A}$ and $\mathrm{C}$, common cold and enteroviruses, and many more, all have RNA genomes. DNA viruses such as herpes viruses, adenoviruses and papillomavirus can cause very serious disease, but other than smallpox, DNA viruses have not historically been associated with mass pandemics that can cause deaths in the millions. Nor do they (or other potential pathogens like bacteria, fungi and parasites) mutate anywhere near as fast as RNA viruses [1], so they tend to be more genetically stable, rather than a moving target for vaccine and antiviral drug design. 
Thus, among the viruses, RNA viruses appear to be particularly well suited as agents of new emerging virus outbreaks and global pandemics, because of several unique characteristics that enable rapid adaptation. First, their very small genome size (typically between 10 and 30 thousand nucleotides) allows for fast replication, easily attaining multiple generations within a 24 hour period [2]. Second, their RNA polymerases are highly error-prone, due to lack of proof-reading ability (with a few notable exception like the nsp14 3'-5' exoribonuclease of coronaviruses and other Nidovirales), so that their mutation rate is not only many orders of magnitude higher $\left(\sim 10^{6}\right)$ than host DNA-based genomes, but is also substantially higher (100-fold or more) than typical DNA viruses [2,3]. This accelerated evolutionary capability enables them to adapt following species transfer, in order to optimize the required host receptor tropism to attain a foothold in the new host population. It also enhances their ability to continuously evade immune surveillance, as illustrated by the need for the production of new seasonal flu vaccines every year. These considerations and more have been succinctly reviewed by Carrasco-Hernandez et al [1], who on this basis (in light of COVID-19), successfully predicted in 2017 that the next global pandemic would involve an RNA virus.

A number of animal RNA viruses transmitted by arthropods, primarily mosquitoes and ticks, have proven to be pathogenic in humans after transfer from another species, whereas there are almost no DNA viruses that infect animals that are known to be arthropod borne, with the notable exception of African Swine Fever Virus, which luckily is not a threat to humans. Many other RNA viruses, like the SARS coronaviruses, influenza and primate immunodeficiency viruses, are directly transmitted between various animal species with varying degrees of ease or difficulty, without the need for a bloodeating insect as an intermediary. The frequency of such inter-animal transmissions is much higher for RNA viruses than for DNA viruses [4].

If the greatest zoonotic and pandemic threats we face are from RNA viruses, to fully understand their pathogenic mechanisms and possible ways to reduce the severity of their impact, we must seek to understand the modi operandi that they have developed as a consequence of their fundamental characteristics as RNA viruses. Of these, none is more fundamental than the simple fact that RNA viruses need the cells they infect to make RNA in copious amounts, to enable the formation of as many viral progeny as the system can bear. Herein, perhaps, lies a vulnerability.

\section{DNA biosynthesis depletes the pool of RNA precursors: a critical role for selenium}

Although new evidence may offer alternatives to the RNA World Hypothesis [5], which posits that DNA evolved later than RNA [6], the fact remains that for all life on earth, DNA biosynthesis is an add-on to RNA biochemistry, so that 2'-deoxyribonucleotides can only be made from ribonucleotides. Hence, DNA synthesis inevitably depletes the pool of ribonucleotide precursors that an RNA virus would need for copying its RNA for new virus production. This means that RNA viruses can increase viral RNA production by partially blocking the synthesis of DNA. There are various ways that they could manage to do that, most of which may be utilized to a varying extent by different RNA viruses. But one of the best ways to slow DNA synthesis involves selenium, and that is the focus of this commentary, as it can help to explain a lot of previous observations about RNA viruses and selenium.

The thioredoxin system is a key redox cycle involved in the reduction of ribose to deoxyribose, in which thioredoxin serves as a hydrogen donor for ribonucleotide reductase (RNR). To sustain that redox cycle, thioredoxin reductase (TR), a selenium-containing enzyme in mammals, is essential. Hence, TR is a perfect target for an RNA virus to slow down DNA synthesis. Specifically, antisense targeting of TR isoforms would be an elegant way for an RNA virus to partially inhibit DNA synthesis to enhance viral RNA synthesis, so that there will be more RNA to make into new viruses. As an 
83

84

85

86

87

88

89

90

91

92

93

94

95

96

97

98

99

100

101

102

103

104

105

106

107

108

109

110

111

112

113

114

115

116

117

118

119

120

121

122

123

124

125

126

essential component of TR, selenium thus could be considered a natural antagonist of RNA viruses, which casts a new light on an extensive body of literature linking selenium status to the incidence, morbidity and mortality of a number of RNA viral infections (as reviewed, [7-9]).

\section{The role of selenium in COVID-19 follows a pattern seen with many RNA viruses}

The recent demonstration by Zhang et al. of a highly significant association between the outcome of SARS-CoV-2 (SCoV2) infection and previously documented regional selenium (Se) status in Chinese cities [10] is just the latest example of a role for selenium that has been reported for a variety of RNA viruses and reverse transcribing viruses with an RNA stage (HIV-1 and Hepatitis B virus) going back four decades. That these cases form a consistent pattern for the involvement of selenium in the incidence, progression or outcome of a variety of viral infections is attested by the fact that over the last several decades, this phenomenon has been the subject of a considerable number of independent reviews, of which I will cite only a few of the most recent [7-9].

In some cases, selenium compounds have been found to have direct antiviral activity either in cell culture (e.g., for influenza and oncogenic retroviruses [11,12]) or in an animal model (e.g., mouse mammary tumor virus, coxsackievirus and influenza [13-15]), or a clinical benefit in a human viral disease, e.g. HIV-1 (as reviewed in [9]) and epidemic hemorrhagic fever linked to hantavirus infection [16]. In other examples, the frequency of cases of infection, viral pathogenicity or disease progression has been found to be associated with either low Se status in patients (HIV-1, influenza), or with a geographic area in which Se deficiency was endemic due to low soil Se content (Coxsackievirus, hepatitis B and hantavirus), as reviewed by various authors [7-9,17]. For the viral infections in each of the latter examples, the increased mortality risk associated with low selenium status or reduced intake in the affected geographic region was significantly reduced by selenium supplementation in every case.

\section{$4 \quad$ The discovery and significance of regions of antisense complementarity between RNA} virus mRNAs and host mRNAs encoding isoforms of thioredoxin reductase (TR)

As my group first reported in regard to HIV-1 and the Zaire Ebolavirus (EBOV) [17], and later for Zika [18], the possibility that those RNA viruses target thioredoxin reductases (TR) by antisense is supported by computational RNA:RNA hybridization results and preliminary experimental data, in the form of gel shift assays with DNA oligonucleotides. We initially discovered those interaction sites in HIV-1 and EBOV because in both cases they were proximal to highly conserved UGA stop codons (potentially encoding selenocysteine) that terminate the HIV-1 nef and EBOV nucleoprotein open reading frames. Although years earlier we had identified (by sequence analysis), cloned and expressed an HIV-1 encoded frameshift variant of the viral gp120 envelope protein and showed that it encoded a functional glutathione peroxidase (GPx, the prototypical selenoprotein), we had to incorporate a mammalian selenocysteine insertion sequence (SECIS) element in the construct in order to express the viral GPx as a selenoprotein [19]. We were never able to identify a functional SECIS element encoded by an RNA virus. Thus, the discovery of the improbable juxtaposition of a highly conserved viral UGA codon with a nearby region of strong antisense complementarity to a host selenoprotein immediately suggested a viral mechanism for capture, by "antisense tethering interactions" (ATI), of a host SECIS element [17]. This mechanism could enable the recoding of the viral UGA stop codon as selenocysteine, to form a low-abundance extended selenoprotein variant of the known viral protein. In retrospect this is not at all surprising, because viruses contain only the barest elements of the machinery of life, primarily what they need to get in and out of cells and to replicate their RNA or DNA; they hijack all the cellular machinery for almost everything else. So it makes sense that HIV and EBOV might also hijack SECIS elements. However, because that capture involved an antisense interaction, there is a 
127 direct implication that this could cause knockdown of host TR1 or TR3 levels as "collateral damage"

128 - but perhaps it isn't collateral damage at all, perhaps it is also deliberately benefiting the virus. And

129 the most obvious benefit would be via the role of TR in DNA synthesis.

130 We have now demonstrated selenium-dependent readthrough of both of those UGA codons, in HIV-1 131 nef and the EBOV nucleoprotein, and a role for TR1 in the mechanism in the case of nef, via GFP 132 reporter gene assays [20,21]. The fact that in database searches these and other RNA virus mRNAs 133 consistently show a preference for antisense targeting of TR over other viral selenoproteins like GPx supports the supposition that the knockdown of the targeted TR isoforms likely to result from such interactions might also benefit an RNA virus, via the role of TR in DNA synthesis [18]. Figure 1 shows computed RNA secondary structure renditions of these and other virus/human RNA:RNA antisense interactions involving either TR1 or TR3 isoforms. To be clear, despite the evidence for seleniumdependent UGA readthrough reviewed above for HIV-1 and EBOV, for the other viruses shown in Figure 1, we have found no evidence that mumps, Zika or influenza A viruses encode selenoprotein modules. Thus, the antisense interactions shown for those viruses may primarily serve to interfere in the synthesis of the targeted isoform of TR, and thereby, DNA synthesis.

\section{Selenium dependence of SARS-CoV-2 outcomes and antisense targeting of TR3}

143 In regard to COVID-19, Zhang et al have shown a remarkable variation in reported outcomes of SCoV2 infection for two regions in China at the extremes of selenium intakes [10]. In Enshi, a city with some of the highest selenium intakes in the world, the reported cure rate for COVID-19 was almost triple the average for all other cities in Hubei Province, including Wuhan. In Hailongjiang, a province in China known for very low levels of selenium, the death rate from COVID-19 was almost 5 times as high as that in all the other provinces outside of Hubei. Both findings were significant at $\mathrm{p}<0.0001$.

This correlation between selenium status and the outcome of yet another RNA virus infection raises the obvious question, could a similar mechanism involving antisense targeting of TR be at work in SCoV2? As shown in Figure 2, a similar analysis identified two SCoV2 regions with antisense matches to human TR3, both having 22 base pairs in a stretch of 23 or 24 nucleotides (equivalent to a high affinity microRNA interaction), with each having only one GU base pair (which are common in RNA helices). The first of these regions (Figure 2A), just before base 5000 in the coronavirus genome, is particularly significant, because it is proximal to a predicted -1 ribosomal frameshift site leading to a region with a single in-frame UGA (potential selenocysteine) codon that is only a few hundred bases upstream from the anti-TR3 antisense site, in the SCoV2 genome of almost 30,000 nucleotides (Supplementary Material Figure S1). Equally compelling is the fact that the targeted site around base 2100 in the human TR3 mRNA is in its 3'-UTR, only 150 bases from the SECIS element that enables the recoding of UGA as selenocysteine; capture of this element is thus a likely factor driving the evolution of this interaction. All of these features were found to be completely conserved in a set of almost 1000 SCoV2 isolates available in Genbank and included in a search on 5-14-2020, with the exception of a few viral isolates which proved to have single-base sequencing misreads (e.g. $\mathrm{N}$ rather than $\mathrm{A}, \mathrm{T}, \mathrm{C}$ or $\mathrm{G}$ ) within this region, contributing to a slightly lower alignment score. Thus, in addition to predicting the knockdown of TR3 mRNA and/or protein levels in SCoV2 infected cells, this example perfectly fulfils the requirements for the viral selenoprotein expression mechanism we proposed for HIV-1 nef and the EBOV nucleoprotein: a $>20$ base long antisense match to a TR isoform within a few hundred bases or less of an accessible in-frame UGA codon [17]. In HIV-1 and EBOV, the nearby UGA codon was accessible as the stop codon of a known gene, enabling an extended protein variant; in

170 SCoV2, the potential coding UGA is accessed via a programmed ribosomal frameshift that was 171 identified by an unbiased algorithm (Figure S1). The targeting of the TR3 isoform by SCoV2 is similar 
172 to what we reported for EBOV, and is also what is computationally predicted for mumps virus (Figure

173 1), whereas HIV-1, influenza and Zika all preferentially target TR1 (Figure 1).

174 TR3 is sometimes called the "testicular" form of TR, because that tissue is where TR3 mRNA levels are highest. But according to the Human Protein Atlas [22], even though mRNA levels are highest in the testes, TR3 protein levels are as high or higher in the lung and GI tract, which are major sites of SCoV2 replication. The Atlas data also show that the ACE2 receptor used by SCoV2 is expressed at high levels in the testes. Significantly, testicular mumps infection has long been known to be a potential complication in males, and in the 2014 EBOV outbreak, cases of persistent EBOV infection of the testes were identified in patients presumed to have recovered [23]. Because of the high levels of ACE2 receptor there, SCoV2 could also target the testes. So all three of these TR3-targeting viruses appear to at least have the potential to infect the tissue in which TR3 is most highly expressed in human males.

The glutaredoxin system and non-selenium dependent inhibition of DNA synthesis

The thioredoxin system seems particularly critical for DNA synthesis in certain cell types and conditions, such as during T cell proliferation [24]. But there is a backup system for DNA synthesis, the glutaredoxin system, which uses glutathione rather than thioredoxin as its hydrogen/electron donor [25]. Significantly, TR3 is unique among TR isoforms in that it contains an N-terminal glutaredoxin domain, so it can function in both the thioredoxin and glutaredoxin systems to sustain DNA synthesis. Thus, antisense-mediated knockdown of TR3 could be an effective general strategy for RNA viruses because of its ability to partially interfere with both redox systems that provide electrons to RNR for reduction of ribonucleotides.

The glutaredoxin system is one of the various non-selenium dependent means mentioned earlier (i.e., not involving TR isoforms), by which an RNA virus could slow down DNA synthesis. Antisense targeting of RNR subunits, or glutaredoxin isoforms, or enzymes involved in glutathione synthesis, could all potentially achieve a similar goal, alone or in combination with anti-TR based mechanisms. Possible examples of these can be found, one of the most convincing being the inhibition of glutathione synthesis by HIV-1, which would inhibit the ability of the glutaredoxin system to provide electrons to RNR. There is an extensive body of evidence dating to the mid-1980s of a progressive deficit of reduced glutathione (GSH) in AIDS patients (reviewed in section 2.1.2. of [26]), and real-time PCR analysis has shown an $89 \%$ knockdown of glutathione synthetase (GSS) in HIV-1 infected macrophages [27]. This may be driven by antisense targeting of GSS mRNA by HIV-1, as suggested by the antisense BLAST hit shown as Figure S2A. Thus HIV-1 may be an example of simultaneous interference in both the thioredoxin system (by TR1 knockdown) and the glutaredoxin system (by GSS knockdown). Simultaneous blockade of both redox systems may prove to be necessary in order to significantly favor RNA synthesis. Significantly, the very large genome size of some DNA viruses, particularly poxviruses, affords them the luxury of encoding their own glutaredoxins, thioredoxin-like proteins, and even RNR homologues [28], which serve in part to facilitate viral DNA synthesis, as well as thiol reduction for viral assembly and other purposes. That pretty much proves the case that viruses can benefit by shifting the RNA:DNA balance in their favor, and that a variety of mechanisms could be used to achieve this goal.

211 In regard to the possible antisense targeting of glutaredoxins by RNA viruses, some of the strongest 212 identifiable matches are between regions of glutaredoxin-2 (GLRX2) and respiratory syncytial viruses (also known as orthopneumovirus Subgroup A), as well as GLRX2 vs. Eastern Equine Encephalitis Virus (EEEV), shown in Figure S2 B-D. It is more difficult to find good examples of potential viral antisense targeting of RNR, which if it exists seems much less common, and the potential interactions 
216

217

218

219

220

221

222

223

224

225

226

227

228

229

230

231

232

233

234

235

236

237

238

239

240

241

242

243

244

245

246

247

248

249

250

251

252

253

254

255

256

257

258

259 less convincing. One possible explanation for this is that, since there is no backup enzyme for RNR, its knockdown could risk shutting down essential DNA repair processes.

Overall, TR isoforms may be ideal targets for RNA viruses because on the one hand, the thioredoxin system appears to be the predominant electron donor for RNR, particularly in the cell cycle $\mathrm{S}$ phase [25], but even if TR1 was totally blocked, the glutaredoxin system assures a basal level of DNA synthesis that may be necessary for continued cell viability. And if the viral agenda also includes the expression of its own selenoprotein module, such as a viral GPx [19,29], antisense targeting of TR isoforms is an ideal choice, because it achieves 2 goals simultaneously, by TR knockdown to increase RNA synthesis, while simultaneously exploiting the ATI mechanism for SECIS capture [17]. This would be very typical of how viruses operate, to do more with less, by encoding multifunctional RNAs and proteins.

\section{$7 \quad$ Discussion and conclusions}

Given the diversity of viruses and possible mechanisms, it is clear that some RNA viruses may interfere in selenium-based mechanisms more than others, and there could even be significant variation in this regard between different subytpes and strains of a given virus. For example, the predicted anti-TR1 interaction shown for a bird flu strain in Figure 1 is an exceptionally strong interaction, not seen at that level of significance for other common strains of influenza A. However, selenium status has been linked in various ways to influenza virus pathogenicity, as recently reviewed [7,9], so the potential role of anti-TR1 interactions in the pathogenesis of influenza merits further investigation. In regard to expected knockdown of TR isoforms by the antisense mechanism, this may occur at the protein level without visible changes in TR mRNA levels. As discussed previously, based on precedents from microRNAs, inhibition of protein synthesis without degradation of the targeted $m R N A$ is actually the expected result if the RNA:RNA base pairing is imperfect, i.e., with more gaps and bulges [30]. However, if the base pairing is almost perfectly continuous, like those predicted for SCoV2 vs. TR3 in Figure 2, it is more likely that knockdown may be observed at both the mRNA and protein levels. But if there are typical structural irregularities in stem regions of the RNA:RNA interaction (as seen for HIV-1:TR-1 in Figure 1), a failure to observe mRNA knockdown via qRT-PCR or microarray does not necessarily rule out this mechanism. This point is validated by the fact that cellular levels of TR1 protein are in fact substantially decreased in HIV-1 infected cells [31] (consistent with our antisense results, Figure 1 and ref. [17]), but TR1 is not a gene that has been reported to be downregulated by HIV-1 at the mRNA level in microarray studies. So this may be a case of antisense disruption of protein synthesis primarily at the ribosomal level.

To summarize the major theme of RNA viruses vs. DNA synthesis as it relates to selenium, the central basis is that in mammals, TR enzymes are selenoproteins, so selenium is an essential component of TR; hence, as part of the thioredoxin system, selenium plays an important role in the eternal competition between DNA and RNA synthesis. This implies that, even in the absence of specific antisense or other targeting of TR by an RNA virus, a more universal sensitivity to selenium status could still exist for this class of viruses. Under conditions of selenium deficiency sufficient to substantially decrease TR protein levels, DNA synthesis may be at least somewhat disfavored, conferring an advantage to RNA viruses. The converse may also be true - that a more replete selenium status may tend to enhance DNA synthesis, creating less favorable conditions for RNA viral replication by depletion of ribonucleotides, thereby providing a protective antiviral benefit.

It should be emphasized, however, that there are a multitude of possible mechanisms by which selenium can influence viral infections, involving both host and viral factors; this just happens to be

This is a provisional file, not the final typeset article 
260

261

262

263

264

265

266

267

268

269

270

271

272

273

274

275

276

277

278

279

280

281

282

283

284

285

286

287

288

289

290

291

292

293

294

295

296

one that particularly applies to RNA viruses as a class. For example, the importance of selenium to the immune system has been reviewed many times (recently, here [32]), and there are specific roles of selenium in human biology that may be relevant to the symptomatology of certain viral infections, e.g. a role in blood clotting, that could be relevant for observed thrombosis in COVID-19, as well as in viral hemorrhagic fevers [9]. The recent identification of human GPx1 as a possible binding partner for the SCoV2 $\mathrm{M}^{\text {pro }}$ protease [33] raises the possibility of host selenoprotein knockdown by proteolysis. Consistent with that possibility, remarkably, there is an instance of an exact match to the SCoV2 $\mathrm{M}^{\text {pro }}$ protease cleavage consensus sequence LQ/A near the very C-terminal of human TR1, which could enable $\mathrm{M}^{\text {pro }}$ to clip off 5 amino acids including the $\mathrm{C}$-terminal redox center of TR1, with the catalytic selenocysteine in the penultimate position. Thus, we may have instances of targeting by SCoV2 of two different isoforms of TR, one by proteolysis (TR1) and one via antisense knockdown (TR3). But the common theme is direct viral interference with the host selenoproteome.

In conclusion, considering the new evidence for a significant correlation between selenium status and reported COVID-19 outcomes [10], and computational evidence presented here for antisense targeting of human TR3 mRNA by SCoV2 (Figure 2), both taken in light of past precedents involving other RNA viral diseases, a call for renewed investigations of the molecular mechanisms involved in what might best be called the "anti-pathogenic" effects of selenium is strongly justified. Rarely has a simple and affordable dietary factor shown such promise to contribute to our ability to withstand an entire class of feared and deadly diseases.

\section{Conflict of Interest}

The author declares that this research was conducted in the absence of any commercial or financial relationships that could be construed as a potential conflict of interest.

\section{$9 \quad$ Author Contributions}

E.W.T. is the sole contributor to the conception, analysis and writing of this work.

\section{Funding}

This work has been supported by a recurring unrestricted gift from the Dr. Arthur and Bonnie Ennis Foundation, Decatur, IL.

\section{Acknowledgments}

The author would like to acknowledge Mr. Calvin J. Taylor for helpful comments on an earlier version of the manuscript, and Dr. Wilson Radding for pointing out the existence of a potential SCoV2 $\mathrm{M}^{\text {pro }}$ protease cleavage site at the C-terminal of human TR1.

\section{Literature Cited}

1. Carrasco-Hernandez, R.; Jacome, R.; Lopez Vidal, Y.; Ponce de Leon, S. Are RNA Viruses Candidate Agents for the Next Global Pandemic? A Review. ILAR journal 2017, 58, 343-358, doi:10.1093/ilar/ilx026.

2. Sanjuan, R.; Nebot, M.R.; Chirico, N.; Mansky, L.M.; Belshaw, R. Viral mutation rates. J Virol 2010, 84, 9733-9748, doi:10.1128/JVI.00694-10. 
304

305

306

307

308

309

310

311

312

313

314

315

316

317

318

319

320

321

322

323

324

325

326

327

328

329

330

331

332

333

334

335

336

337

338

339

340

3. Peck, K.M.; Lauring, A.S. Complexities of Viral Mutation Rates. J Virol 2018, 92, doi:10.1128/JVI.01031-17.

4. Wells, K.; Morand, S.; Wardeh, M.; Baylis, M. Distinct spread of DNA and RNA viruses among mammals amid prominent role of domestic species. Glob Ecol Biogeogr 2020, 29, 470-481, doi:10.1111/geb.13045.

5. Xu, J.; Chmela, V.; Green, N.; Russell, D.; Janicki, M.; Gora, R.; Szabla, R.; Bond, A.; Sutherland, J. Selective prebiotic formation of RNA pyrimidine and DNA purine nucleosides. Nature 2020, 582, 6066.

6. Gilbert, W. The RNA World. Nature 1986, 319, 618.

7. Steinbrenner, H.; Al-Quraishy, S.; Dkhil, M.A.; Wunderlich, F.; Sies, H. Dietary Selenium in Adjuvant Therapy of Viral and Bacterial Infections. Adv Nutr 2015, 6, 73-82, doi:10.3945/an.114.007575.

8. Guillin, O.M.; Vindry, C.; Ohlmann, T.; Chavatte, L. Selenium, Selenoproteins and Viral Infection. Nutrients 2019, 11, doi:10.3390/nu11092101.

9. Hiffler, L. Selenium and RNA viruses interactions: Potential implications for SARS-Cov-2 infection (Covid-19). OSF Preprints, 2020; 10.31219/osf.io/vaqz6.

10. Zhang, J.; Taylor, E.W.; Bennett, K.; Saad, R.; Rayman, M.P. Association between regional selenium status and reported outcome of COVID-19 cases in China. Am J Clin Nutr 2020, doi:10.1093/ajcn/nqaa095.

11. Lazymova, Z.A.; Abdullaev, II; Abdullaev, F.I.; Asadullaev, T.A. [Inhibiting action of sodium selenite on the reproduction of the influenza virus]. Voprosy virusol 1986, 31, 236-238.

12. Balansky, R.M.; Argirova, R.M. Sodium selenite inhibition of the reproduction of some oncogenic RNA-viruses. Experientia 1981, 37, 1194-1195, doi:10.1007/BF01989914.

13. Schrauzer, G.N.; Molenaar, T.; Kuehn, K.; Waller, D. Effect of simulated American, Bulgarian, and Japanese human diets and of selenium supplementation on the incidence of virally induced mammary tumors in female mice. Biol Trace Elem Res 1989, 20, 169-178.

14. Beck, M.A.; Kolbeck, P.C.; Shi, Q.; Rohr, L.H.; Morris, V.C.; Levander, O.A. Increased virulence of a human enterovirus (coxsackievirus B3) in selenium-deficient mice. J Infect Dis 1994, 170, 351-357.

15. Beck, M.A.; Nelson, H.K.; Shi, Q.; Van Dael, P.; Schiffrin, E.J.; Blum, S.; Barclay, D.; Levander, O.A. Selenium deficiency increases the pathology of an influenza virus infection. FASEB J 2001, 15, 1481-1483.

16. Hou, J.C. Inhibitory effect of selenite and other antioxidants on complement-mediated tissue injury in patients with epidemic hemorrhagic fever. Biol Trace Elem Res 1997, 56, 125-130, doi:10.1007/BF02778988.

17. Taylor, E.W.; Ruzicka, J.A.; Premadasa, L.; Zhao, L. Cellular selenoprotein mRNA tethering via antisense interactions with Ebola and HIV-1 mRNAs may impact host selenium biochemistry. Curr Top Med Chem 2016, 16, 1530-1535, doi:10.2174/1568026615666150915121633.

18. Taylor, E.W.; Ruzicka, J.A. Zika-mediated antisense inhibition of selenoprotein synthesis may contribute to neurologic disorders and microcephaly by mimicking SePP1 knockout and the genetic disease PCCA. Zika Open Preprint Server, Bull. World Health Organ. 2016, E-pub, 13 July, doi:http://dx.doi.org/10.2471/BLT.16.182071.

19. Zhao, L.; Cox, A.G.; Ruzicka, J.A.; Bhat, A.A.; Zhang, W.; Taylor, E.W. Molecular modeling and in vitro activity of an HIV-1-encoded glutathione peroxidase. Proc Natl Acad Sci U S A 2000, 97, 63566361, doi:10.1073/pnas.97.12.6356. 
341

342

343

344

345

346

347

348

349

350

351

352

353

354

355

356

357

358

359

360

361

362

363

364

365

366

367

368

369

370

371

372

373

374

375

376

377

378

379

380

381

382

383

384

20. Taylor, E.W.; Ruzicka, J.A.; Premadasa, L. Translational readthrough of the Ebola nucleoprotein 3'UGA codon via antisense tethering of thioredoxin reductase 3 mRNA. Presented at the International Congress on Targeting Ebola, Paris, France, 28-29 May, 2015, doi:10.13140/RG.2.2.10237.51683.

21. Premadasa, L.S.; Dailey, G.P.; Ruzicka, J.A.; Taylor, E.W. Selenium-dependent readthrough of the conserved 3'-terminal UGA stop codon of HIV-1 nef. Preprints.org 2020, doi:10.20944/preprints202005.0432.v1.

22. Uhlen, M. Tissue-based map of the human proteome. Science 2015, 347, 6220, 1260419 https://www.proteinatlas.org/ENSG00000197763-TXNRD00000197763/tissue.

23. Schindell, B.G.; Webb, A.L.; Kindrachuk, J. Persistence and Sexual Transmission of Filoviruses. Viruses 2018, 10, doi:10.3390/v10120683.

24. Muri, J.; Heer, S.; Matsushita, M.; Pohlmeier, L.; Tortola, L.; Fuhrer, T.; Conrad, M.; Zamboni, N.; Kisielow, J.; Kopf, M. The thioredoxin-1 system is essential for fueling DNA synthesis during T-cell metabolic reprogramming and proliferation. Nature Comm 2018, 9, 1851, doi:10.1038/s41467-01804274-w.

25. Zahedi Avval, F.; Holmgren, A. Molecular mechanisms of thioredoxin and glutaredoxin as hydrogen donors for Mammalian s phase ribonucleotide reductase. J Biol Chem 2009, 284, 8233-8240, doi:10.1074/jbc.M809338200.

26. Taylor, E.W. The oxidative stress-induced niacin sink (OSINS) model for HIV pathogenesis. Toxicology 2010, 278, 124-130, doi:10.1016/j.tox.2009.10.018.

27. Morris, D.; Guerra, C.; Donohue, C.; Oh, H.; Khurasany, M.; Venketaraman, V. Unveiling the mechanisms for decreased glutathione in individuals with HIV infection. Clin Dev Immunol 2012, 2012, 734125, doi:10.1155/2012/734125.

28. Gubser, C.; Hue, S.; Kellam, P.; Smith, G.L. Poxvirus genomes: a phylogenetic analysis. J Gen Virol 2004, 85, 105-117, doi:10.1099/vir.0.19565-0.

29. Zhang, W.; Ramanathan, C.S.; Nadimpalli, R.G.; Bhat, A.A.; Cox, A.G.; Taylor, E.W. Seleniumdependent glutathione peroxidase modules encoded by RNA viruses. Biol Trace Elem Res 1999, 70, 97-116, doi:10.1007/BF02783852.

30. Zeng, Y.; Yi, R.; Cullen, B.R. MicroRNAs and small interfering RNAs can inhibit mRNA expression by similar mechanisms. Proc Natl Acad Sci U S A 2003, 100, 9779-9784, doi:10.1073/pnas.1630797100.

31. Gladyshev, V.N.; Stadtman, T.C.; Hatfield, D.L.; Jeang, K.T. Levels of major selenoproteins in T cells decrease during HIV infection and low molecular mass selenium compounds increase. Proc Natl Acad Sci U S A 1999, 96, 835-839, doi:10.1073/pnas.96.3.835.

32. Avery, J.C.; Hoffmann, P.R. Selenium, Selenoproteins, and Immunity. Nutrients 2018, 10, doi:10.3390/nu10091203.

33. Gordon, D.E.; Jang, G.M.; Bouhaddou, M.; Xu, J.; Obernier, K.; White, K.M.; O'Meara, M.J.; Rezelj, V.V.; Guo, J.Z.; Swaney, D.L., et al. A SARS-CoV-2 protein interaction map reveals targets for drug repurposing. Nature 2020, 10.1038/s41586-020-2286-9, doi:10.1038/s41586-020-2286-9.

34. Rehmsmeier, M.; Steffen, P.; Hochsmann, M.; Giegerich, R. Fast and effective prediction of microRNA/target duplexes. RNA 2004, 10, 1507-1517, doi:10.1261/rna.5248604.

35. Wright, P.R.; Georg, J.; Mann, M.; Sorescu, D.A.; Richter, A.S.; Lott, S.; Kleinkauf, R.; Hess, W.R.; Backofen, R. CopraRNA and IntaRNA: predicting small RNA targets, networks and interaction domains. Nucleic Acids Res 2014, 42, W119-123, doi:10.1093/nar/gku359. 


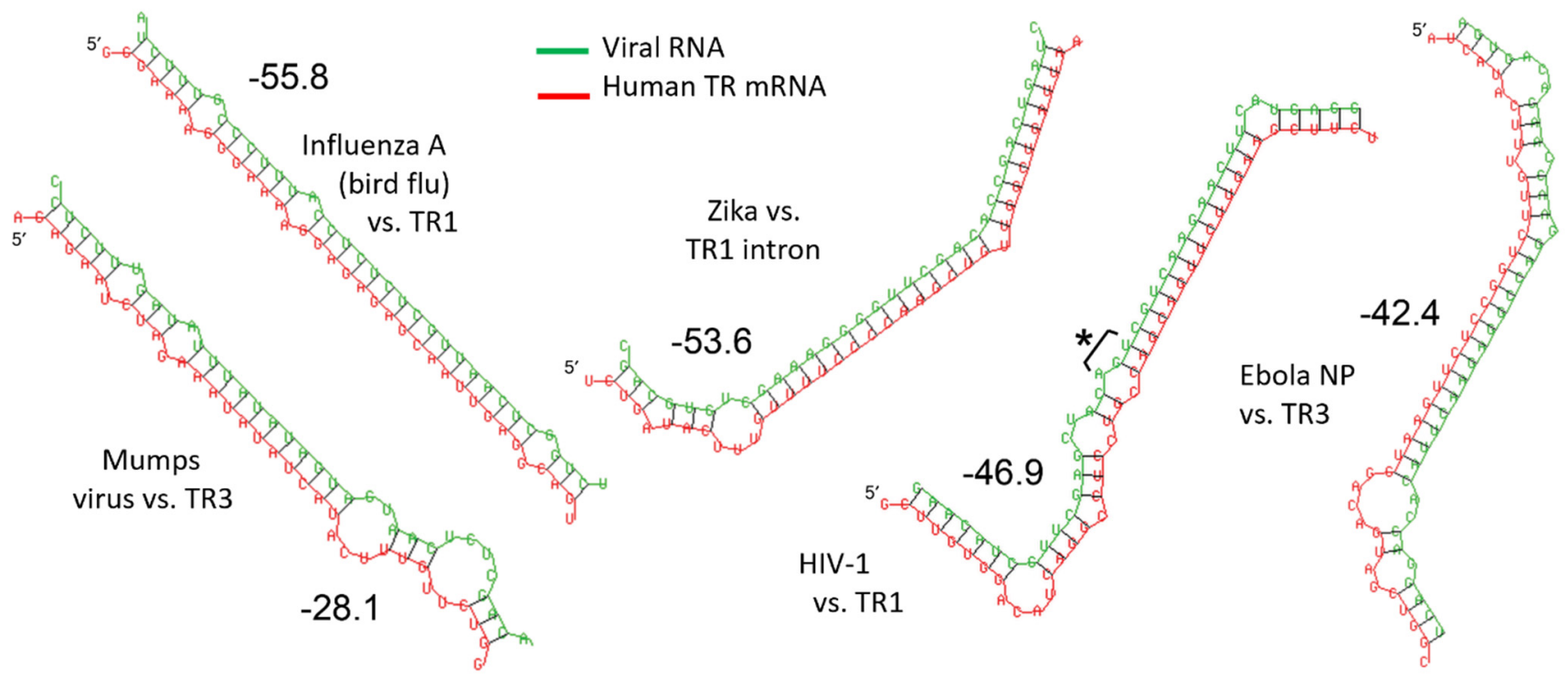

385 Figure 1. Predicted antisense interactions for various RNA viruses targeting human TR isoforms. These include previously published interactions for the EBOV nucleoprotein mRNA vs TR3, the HIV1 nef 3 ' region vs TR1, and Zika mRNA vs. TR1 [17,18]. The asterisk indicates the 3'-UGA stop codon of HIV-1 nef, where selenium-dependent readthrough occurs [21]. Additional predicted interactions with either TR1 or TR3 are shown for a strain of avian influenza and mumps virus. All of these interactions were initially identified as DNA/DNA +/- matches using BLAST, then confirmed at the RNA level using the RNAHybrid program [34], and finally confirmed to be sufficiently strong as to overcome internal folding energies of the individual RNA strands using the IntaRNA program [35], as described previously [17]. The Genbank accession numbers and regions for the sequence fragments shown are given in the relevant references, the others are: Bird flu vs human TR 1: the antisense match is between the genomic negative sense strand of H9N2 Influenza A virus (A/duck/Nanjing/2/97) nonstructural protein 1 (Genbank DQ064482, bases 710-682) and human TR1 (Genbank NM_003330.4, bases 3484-3518). Mumps virus vs. TR3: Mumps virus (Genbank NC_002200, 1062510659) vs human TR3 (Genbank NM_052883.2, 1754-1787). 


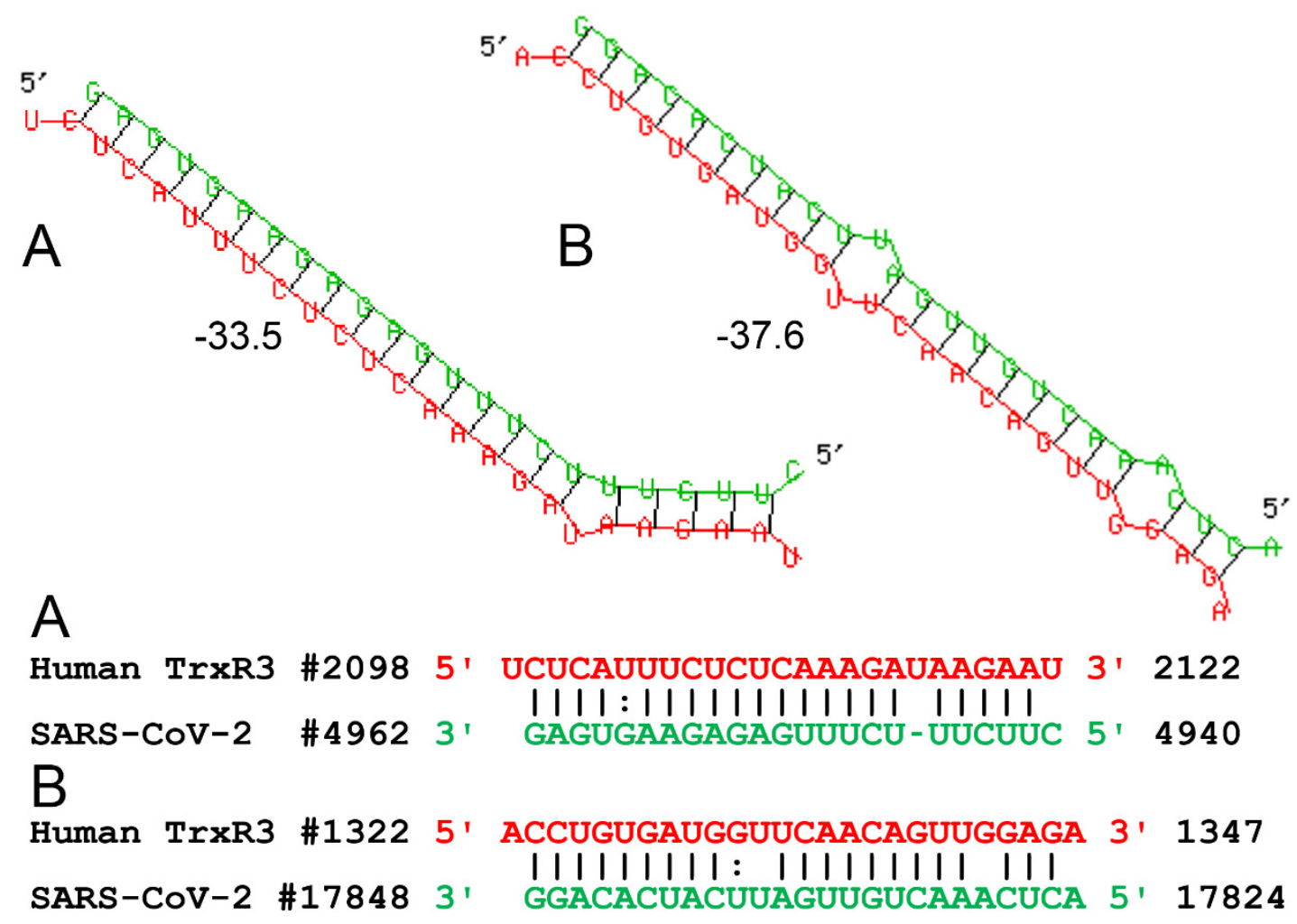

399 Figure 2. Predicted RNA:RNA antisense interactions between SARS-CoV-2 and human 400 thioredoxin reductase 3 (TrxR3) mRNAs. Two potential interaction sites, $\mathbf{A}$ and $\mathbf{B}$, were identified 401 using procedures described previously [17]. Numbering for the locations of each fragment correspond 402 to Genbank reference sequences NC_045512 (SCoV2) and NM_052883.2 (TrxR3, TR3). The RNA 403 secondary structures shown and the computed interaction free energies in $\mathrm{kcal} / \mathrm{mol}$ (numerals next to 404 the structures) were generated using the RNAHybrid 2.2 program [34]. These results suggest that the 405 resulting knockdown of TR3 may contribute to the pathology and selenium-dependent outcome of 406 COVID-19 [10].

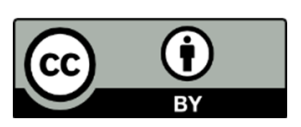

(C) 2020 by the authors. Submitted for possible open access publication under the terms and conditions of the Creative Commons Attribution (CC BY) license (http://creativecommons.org/licenses/by/4.0/). 